\title{
Die Bedeutung beruflicher Erfahrungen und fachsystematischen Wissens für die Bewältigung fachlicher Problemstellungen - eine Analyse bei angehenden Technikern und Technikerinnen
}

\author{
Alexander Nitzschke (D) - Stefanie Velten - Agnes Dietzen • \\ Reinhold Nickolaus
}

Eingegangen: 27. Juli 2018 / Angenommen: 6. März 2019 / Online publiziert: 25. März 2019

(C) Der/die Autor(en) 2019

Zusammenfassung Im Bereich der beruflichen Kompetenzdiagnostik zeigt sich, dass vor allem kognitive Faktoren eine zentrale Rolle bei der Erklärung der erreichten Leistungsniveaus einnehmen. Berufliche Erfahrungen werden hingegen nur zum Teil als relevant ausgewiesen und bleiben in ihrer Erklärungskraft zumeist hinter den kognitiven Faktoren zurück. Maßgeblich hierbei scheint unter anderem die Operationalisierung beruflicher Erfahrungen, wie beispielsweise über die Dauer der Tätigkeit oder über Selbsteinschätzungen der Befragten. Der Beitrag geht der Frage nach, welcher Einfluss den beruflichen Erfahrungen neben den kognitiven Faktoren zukommt. In der Untersuchung, welche 272 bzw. 208 angehende staatlich geprüfte Techniker und Technikerinnen der Elektrotechnik erfasste, wurde die berufliche Erfahrung einerseits über die Dauer der Berufsausübung nach Erwerb der Facharbeiterqualifikation und andererseits über die Häufigkeit der Durchführung berufstypischer Tätigkeiten im Rahmen der praktischen Berufsausübung erfasst. Es zeigt sich, dass unter Kontrolle der kognitiven Variablen (kognitive Grundfertigkeit und fachspezifisches Wissen) die Dauer der Berufsausübung keine zusätzliche prädiktive Kraft zur Erklärung der Fachkompetenz erbringt. Dahingegen erbringt die Operationalisierung der Berufserfahrung auf Basis konkreter Tätigkeiten, welche

\footnotetext{
A. Nitzschke $(\bowtie) \cdot$ Prof. Dr. R. Nickolaus Universität Stuttgart, Stuttgart, Deutschland E-Mail: nitzschke@bwt.uni-stuttgart.de

Prof. Dr. R. Nickolaus

E-Mail: nickolaus@bwt.uni-stuttgart.de

S. Velten · Dr. A. Dietzen

Bundesinstitut für Berufsbildung (BIBB), Bonn, Deutschland

S. Velten

E-Mail: velten@bibb.de

Dr. A. Dietzen

E-Mail: dietzen@bibb.de
} 
zu Tätigkeitsbündeln zusammengefasst wurden, einen deutlichen und signifikanten Beitrag zur Erklärung der berufsfachlichen Leistung.

Schlüsselwörter Berufliche Fortbildung · Fachwissen · Fachspezifische Problemlösefähigkeit · Berufliche Erfahrung

\title{
Relevance of professional experience and vocational knowledge for solving problems - an analysis of perspective electrical engineering technicians
}

\begin{abstract}
Research on vocational competence has presented ample evidence that cognitive factors usually are the most powerful predictors of achievement, whereas professional experience often does not add explanatory power to a model and if so, usually to a smaller extent. A potential reason for this finding might be related to how professional experience is measured; common measurements in use are the number of years of work experience on the job or participants' self-assessments on their level of experience. The following article aims at comparing the predictive power of cognitive factors and professional experience, using two different measures for the latter: (1) number of years of work experience after completing the apprenticeship and (2) the actual frequency of carrying out tasks or activities that are considered typical for the profession. The sample for this analysis includes between 272 and 208 persons enrolled in an education program for electrical engineering technicians. Results show that both cognitive variables (here IQ and domain-specific knowledge) and professional experience significantly contribute to explaining professional achievement albeit only when professional experience is measured by taking into account the actual activities (here combined into different groups of activities) carried out by the participants.
\end{abstract}

Keywords Post-secondary vocational educational training · Vocational knowledge · Domain-specific problem-solving skills · Professional experience

\section{Ausgangssituation und Forschungsstand}

Der Fokus von Forschungsarbeiten zur beruflichen Kompetenzdiagnostik liegt bisher im Bereich der dualen Erstausbildung, wohingegen die berufliche Aufstiegsfortbildung bislang wenig Aufmerksamkeit erhält. In diese münden Personen mit stark unterschiedlichen Bildungsbiographien ein, was sich bspw. anhand der Bandbreite unterschiedlicher Lebensphasen und beruflichen Stationen mit den damit einhergehenden beruflichen Erfahrungen zeigt. Um die unterschiedlichen Vorwissensstände adäquat im Fortbildungsverlauf aufzugreifen, ist es nötig, die individuellen berufsfachlichen Kompetenzen zu berücksichtigen. In diesem Zusammenhang ist zu klären, welche Relevanz berufliche Erfahrungen im Rahmen der Leistungsfeststellung einnehmen.

Zur Bedeutung beruflicher Erfahrungen für die berufliche Leistungsfähigkeit liegen sehr unterschiedliche Einschätzungen vor. Einerseits wird unterstellt, dass sich 
die beruflichen Handlungskompetenzen vor allem durch die Erfahrungen am Arbeitsplatz aufbauen (z.B. Geithner und Moser 2009) und andererseits wird in vorliegenden Metastudien der beruflichen Erfahrung z.T. nur eine geringe Erklärungskraft für berufliche Leistungen zugesprochen (Schmidt und Hunter 1998). Deutlich höhere Erklärungskraft für berufsfachliche Leistungen wird in den Metastudien dem Fachwissen bescheinigt (Dye et al. 1993). Daneben zeigen Studien, dass die Erklärungskraft der beruflichen Erfahrungen je nach verwendetem Berufserfolgskriterium unterschiedlich ausfällt. So dokumentieren z. B. $\mathrm{Ng}$ et al. (2005) für eine Gesamtstichprobe von mehr als 10.000 Personen einen Zusammenhang von $r=0,27$ zwischen Berufserfahrung und Einkommen, ein deutlich geringerer Zusammenhang wird hingegen an gleicher Stelle für den Zusammenhang zwischen Berufserfahrung und Beförderung $(r=0,06)$ auf Basis einer etwas kleineren $(N=5.400)$ Stichprobe berichtet.

Geithner und Moser (2009, S. 71) nehmen an, Berufserfahrung sei eine zentrale Determinante des beruflichen Erfolgs und je länger eine Person in einem bestimmten Tätigkeitsbereich arbeite, desto erfolgreicher sei sie aufgrund ihres Erfahrungsschatzes und den durch Berufserfahrung entwickelten Kompetenzen, auf die sie zurückgreifen könne. Von Bedeutung seien für die beruflichen Leistungen das spezifische Wissen, die Fähigkeiten und Fertigkeiten, die durch den Berufsalltag und die damit verbundenen Herausforderungen entwickelt werden (ebd.). D.h. neben der Dauer der Tätigkeit wird unterstellt, dass die im Arbeitskontext erworbenen Kompetenzen, die wiederum von den Tätigkeitsanforderungen abhängig sind, für die erbrachte Leistung zentral seien. Damit wird neben variierenden Indikatoren für die Erfassung beruflicher Leistungen auf unterschiedliche Faktoren verwiesen, die bei der Modellierung der Berufserfahrung (Dauer der Tätigkeit vs. konkrete Tätigkeitsanforderungen) zu berücksichtigen sind. Darauf verweisen auch Quiñones et al. (1995), die Zusammenhänge von $r=0,43$ zwischen häufigkeitsbasiert erfassten beruflichen Erfahrungen bzw. Tätigkeiten und beruflicher Leistung dokumentieren, im Vergleich $\mathrm{zu} \mathrm{r}=0,27$ für den Zusammenhang zwischen einer rein zeitbasierten Messung beruflicher Erfahrung und beruflicher Leistung. Gleichzeitig berichten Quiñones et al. (1995) auch Vorteile einer tätigkeitsbasierten Erfassung von Erfahrungen bezogen auf Zusammenhänge zwischen Job basiert erhobenen Erfahrungen ${ }^{1}$ und organisationsbasiert erhobenen Erfahrungen ${ }^{2}$ (Zusammenhang von $r=0,27$ auf Jobebene und $r=0,16$ für Zusammenhänge auf Organisationsebene). Die Autoren vermuten, dass ,amount and task-level measures are perhaps better measures of what individuals actually do on the job“ (Quiñones et al. 1995, S. 904). Vor diesem Hintergrund kann unterstellt werden, dass eine eher tätigkeitsbezogene Erfassung der gesammelten Erfahrungen eine stärkere prädiktive Kraft entfaltet als eine Modellierung der Berufserfahrung allein über die Tätigkeitsdauer. Zu klären wäre, ob auch unter

\footnotetext{
${ }^{1}$ Quiñones et al. (1995) modellieren berufliche Erfahrungen zweidimensional über den Grad der Spezifizierung (Tätigkeit, zu Jobs gebündelte Tätigkeiten, organisatorische Kontexte, in welchen die Tätigkeiten erbracht werden) und die zweite Dimension gegliedert in die Menge, zeitliche Ausdehnung und den Typus bzw. den Anspruchsgrad der Tätigkeiten. Jobs werden als aggregierte Tätigkeitsbündel, die sich nach Seniorität und Komplexität unterscheiden können, gefasst.

2 Erhoben über die Anzahl der Organisationen, in welchen man tätig war, die Seniorität und die Organisationstypik (Quiñones et al. 1995).
} 
dieser Bedingung das (primär in formellen Bildungsprozessen erworbene) Fachwissen einen höheren Erklärungsbeitrag für die berufliche Leistung erbringt als die berufliche Erfahrung.

Geithner und Moser (2009) machen auch darauf aufmerksam, dass sich Erfahrungen und der daraus folgende Wissenserwerb nicht linear über den Zeitverlauf verteilen (müssen), sondern insbesondere zu Beginn einer beruflichen Tätigkeit besonders hoch sein können. Bezogen auf die im Folgenden vorgestellte Studie scheint dies insoweit relevant, als die Probanden und Probandinnen aufgrund der Einmündungsbedingungen in die Fortbildung zum staatlich geprüften Techniker bzw. zur staatlich geprüften Technikerin berufliche Erfahrungen im Umfang von mindestens einem Jahr nachweisen müssen und in der Regel eher noch am Anfang ihrer beruflichen Karriere stehen. Mit Varianzen der Erklärungskraft beruflicher Erfahrungen für die Erbringung beruflicher Leistungen ist im Anschluss an Tesluk und Jacobs (1998, S. 333) auch zu rechnen, da sich Menschen dahingehend unterscheiden, ob und wie stark sie aus den gemachten Erfahrungen lernen. Verwiesen wird in diesem Kontext darauf, dass ,learning does not often automatically follow from experience; it requires reflection and a desire to learn from what has occurred as well as an environment that provides opportunities for reflection and learning“" (Tesluk und Jacobs 1998, S. 333).

Variantenreich und herausfordernd scheint in den bisher vorliegenden Studien auch die Erfassung des beruflichen Erfolgskriteriums. Nach Dette et al. (2004) kann beim Konzept des Berufserfolgs zunächst zwischen beruflichem Erfolg in Bezug auf die globale Laufbahn oder in Bezug auf die spezifische Arbeit unterschieden werden. Für beide Bereiche kann Berufserfolg auf der Datenbasis von neutralen Kennzahlen, wie z. B. Gehalt oder konkret zählbaren Leistungen wie produzierte Stückzahlen, auf der Basis von selbsteingeschätzten Zufriedenheitsmaßen sowie durch eigene (z.B. Selbsteinschätzung der beruflichen Leistung) oder durch andere durchgeführte Vergleiche (z.B. Leistungsbeurteilung durch Vorgesetzte oder Kollegen) erfolgen. Unberücksichtigt bleiben in dieser Modellierung berufsfachlicher Leistungen fachliche Kompetenztests, die den Vorteil bieten objektive Vergleichsmaße bereitzustellen, gleichzeitig aber auch vor der Herausforderung stehen, hinreichend reliabel und valide zu sein. Sonnentag et al. (2008) bringen Maße ins Spiel, die sich auf das flexible Verhalten der Mitarbeiter und Mitarbeiterinnen beziehen und damit das Bewältigen von Anforderungen wie Krisen- und Stressmanagement oder Problemlösen umfassen oder auch interkulturelle und interpersonelle Anpassungsleistungen adressieren. Fachliche Problemlöseleistungen ${ }^{3}$ erweisen sich in elektrotechnischen Domänen als besonders leistungskritisch, da ausfallende technische Einheiten zu Produktionsstillständen führen können und damit eine schnelle Behebung von Störungen besonders wichtig wird. Abhängig erweisen sich solche Problemlöseleistungen in hohem Grade von fachlichem Wissen (im Überblick Nickolaus und Seeber 2013), für das seinerseits in Metastudien (Hunter und Hunter 1984; Dye et al. 1993) substantielle Zusam-

\footnotetext{
3 Unterschieden werden in elektrotechnischen Domänen eine analytische und eine konstruktive Subdimension der fachlichen Problemlösekompetenz. Operationalisiert werden analytische Problemlösekompetenzen über die Fähigkeit, in elektrotechnischen Systemen Fehler zu diagnostizieren, und konstruktive z. B. über die Fähigkeit, Steuerungen zu programmieren (vgl. Walker et al. 2015).
} 
menhänge mit beruflichen Leistungen berichtet werden. So dokumentierten Hunter und Hunter (1984) sowie Dye et al. (1993) jeweils auf der Basis großer Fallzahlen Zusammenhänge zwischen berufsfachlichem Wissen und beruflichen Leistungen in der Größenordnung von 0,45. Inzwischen sind die Arbeiten zur Kompetenzdiagnostik in elektrotechnischen Domänen weiter vorangeschritten und ermöglichen neben der objektiven und validen Erfassung des Fachwissens auch die valide Erfassung berufsfachlicher Problemlöseleistungen (vgl. z. B. Walker et al. 2015). Während das Fachwissen über schriftliche Tests erhoben wird, finden für die Erfassung der berufsfachlichen Problemlöseleistungen authentische Simulationen Verwendung, für die gezeigt werden konnte, dass sie gemessen an realen Anforderungssituationen valide Abschätzungen der Kompetenzen erlauben (Abele et al. 2015; Walker et al. 2015). Damit besteht die Möglichkeit, berufsfachliche Leistungen auch bezogen auf Problemlöseleistungen objektiv und valide zu erfassen, was im Vergleich zu den ansonsten meist verwendeten Fremdzuschreibungen ${ }^{4}$ präzisere Schätzungen ermöglicht und damit für die Effektabschätzung von Einflussfaktoren eine bessere Basis darstellt als weniger valide Verfahren wie Selbst- und Fremdeinschätzungen. In den elektrotechnischen Domänen dokumentieren die Arbeiten zur Kompetenzmodellierung sowohl für das Fachwissen als auch die berufsfachliche Problemlösekompetenz Ausdifferenzierungen, die bei der Einlösung des Anspruchs einer validen Erfassung zu berücksichtigen wären. Unterschieden werden bezogen auf das Fachwissen bei Elektronikern und Elektronikerinnen für Automatisierungstechnik sowie für Energie- und Gebäudetechnik jeweils dreidimensionale Fachwissensstrukturen, für die Problemlöseleistungen liegt für die Elektroniker und Elektronikerinnen für Automatisierungstechnik eine Ausdifferenzierung in eine analytische und eine konstruktive Subdimension vor. Das Fachwissen wurde in die Subdimensionen „Grundlagen der Elektrotechnik“, ,elektrische Anlagen und Systeme“ und „Steuerungstechnik“ ausdifferenziert, für die van Waveren und Nickolaus (2016) zeigen, dass die Grundlagen vor allem über schulische Lernprozesse aufgebaut werden, bei den beiden anderen Dimensionen hingegen auch betriebliche Lernerfahrungen, d.h. erfahrungsbasiertes Lernen für den Wissensaufbau bedeutsam werden. Die Zusammenhänge zwischen den einzelnen Fachwissensdimensionen erreichen bei den Elektronikern und Elektronikerinnen für Automatisierungstechnik am Ende der Ausbildung Größenordnungen von 0,74 bis 0,83 (van Waveren und Nickolaus 2015). Offen ist die Frage, ob es im Verlauf der beruflichen Tätigkeit und der Fortbildung verstärkt zu integrativen Verarbeitungsprozessen des Fachwissens kommt. Plausibel scheint dies zumindest für die systematische Fortbildungsphase, in der eine tiefere Verarbeitung des Fachwissens angestrebt wird.

In der im Folgenden vorgestellten Studie, die mit (angehenden) staatlich geprüften Technikern und Technikerinnen der Elektrotechnik im Verlauf der Fortbildung durchgeführt wurde, wurde der Anspruch auf eine testbasierte, problembezogene Leistungserfassung im Rückgriff auf einen fachlichen Problemlösetest eingelöst, für den an anderer Stelle gezeigt werden konnte, dass er die analytische Problemlösekompetenz objektiv, reliabel und valide abbilden kann (Walker et al. 2016). Zugleich

\footnotetext{
${ }^{4}$ Bei Fremdzuschreibungen ist die Güte stark abhängig von der Differenziertheit der Erfassung (Schrader 2012), die Korrelationen mit testbasierten Daten variieren in der Größenordnung von 0,3 und 0,6.
} 
wurden die beruflichen Erfahrungen nicht nur über die Tätigkeitsdauer, sondern inhaltsbezogen erfasst, so dass belastbare Aussagen zur Erklärungskraft der Tätigkeitserfahrungen erwartet werden können. Unberücksichtigt blieben konstruktive berufsfachliche Problemlöseleistungen, die für Elektroniker und Elektronikerinnen für Automatisierungstechnik als eigene allerdings relativ eng mit der analytischen Problemlösekompetenz assoziierte Kompetenzdimension bestätigt werden konnte (Walker et al. 2015).

\section{Fragestellungen und Hypothesen}

In Anbetracht der fortschreitenden Technisierung besteht ein starker Bedarf an ausgebildeten Fachkräften. Neben den Hochschulen und Berufsakademien bilden die Fachschulen eine dritte Säule innerhalb des tertiären Bildungsbereichs, in welchen hoch qualifizierte Fachkräfte im technischen Bereich ausgebildet werden. Die Fortbildung ist hierbei primär an Personen auf Facharbeiterniveau adressiert und stellt für diese eine wichtige Möglichkeit zur Höherqualifikation ausgehend vom System der dualen Berufsausbildung dar.

Mit der vorgelegten Studie soll unter anderem der Frage nachgegangen werden, welche prädiktive Kraft den beruflichen Erfahrungen für das berufsfachliche Wissen und die berufsfachlichen analytischen Problemlöseleistungen zukommt. Dabei soll explizit auch der Frage nachgegangen werden, inwieweit berufliche Erfahrungen, die tätigkeitsbasiert erhoben werden, neben dem Fachwissen geeignet sind, die berufsfachliche Problemlöseleistung vorherzusagen. Des Weiteren soll geklärt werden, ob es im Verlauf der Fortbildung zu einer integrativen Verarbeitung des Fachwissens kommt, was in einer engeren Assoziation der Subdimensionen am Ende gegenüber dem Anfang der Fortbildung Ausdruck finden müsste. Relevant ist die zunehmend integrative Verarbeitung des fachsystematischen Wissens u.a. für dessen Anwendungsfähigkeit, da für stärker vernetztes Wissen eine flexiblere Aktualisierung in unterschiedlichen Anforderungssituationen unterstellt werden kann.

Im Anschluss an den Forschungsstand werden folgende Hypothesen geprüft:

H1: Die einschlägigen beruflichen tätigkeitsbasierten Erfahrungen werden für den Aufbau des Fachwissens bedeutsam, wobei die Erklärungskraft für die einzelnen Subdimensionen des Fachwissens unterschiedlich ausfällt. Erwartet wird, dass der Zusammenhang der beruflichen Erfahrungen mit dem Grundlagenwissen geringer ausfällt als mit den stärker anwendungsbezogenen Subdimensionen des Fachwissens.

H2: Die beruflichen Erfahrungen tragen auch bei Kontrolle des Fachwissens und der kognitiven Grundfertigkeit zusätzlich zur Erklärung der berufsfachlichen Problemlöseleistungen bei.

H3: Die Subdimensionen des Fachwissens korrelieren zum Ende der Fortbildung höher als zu deren Beginn. 


\section{Anlage der Studie}

Die Studie wurde im Rahmen der Fortbildung zum staatlich geprüften Techniker bzw. zur staatlich geprüften Technikerin der Fachrichtung Elektrotechnik an Fachschulen durchgeführt und war längsschnittlich angelegt. Einbezogen wurden Fortbildungsschülerinnen und -schüler innerhalb einer zweijährigen Fortbildung in Vollzeit, welche sich in etwa zu gleichen Teilen auf die Bundesländer Baden-Württemberg und Nordrhein-Westfalen verteilen. In der Eingangsmessung (Herbst 2015/Frühjahr 2016) konnten Daten von 272 Testpersonen erfasst werden, im Abschlusstest, der Ende 2016 bzw. Anfang 2017 erfolgte, wurden noch 208 Testpersonen erreicht. Das Alter der Testpersonen lag zu Beginn der Fortbildung im Mittel bei 25,4 Jahren (SD 4,7). Bei den formalen allgemeinen Schulabschlüssen überwiegt die Gruppe mit mittlerem Abschluss (64\%), der Anteil mit Hochschulzugangsberechtigung liegt bei $19 \%, 5 \%$ verfügten über einen Hauptschulabschluss, der Rest machte keine Angaben.

Bemerkenswert war die hohe Heterogenität der Teilnehmenden, sowohl gemessen an den formalen Abschlüssen, die verschiedene Handwerksberufe und auch Industrieberufe abdeckten, als auch gemessen an den kognitiven Eingangsvoraussetzungen und den beruflichen Erfahrungen (Nitzschke et al. 2017). Größere Anteile der Stichprobe hatten Abschlüsse in Industrieberufen wie z.B. Elektroniker und Elektronikerin für Betriebstechnik, Automatisierungstechnik oder Mechatroniker bzw. Mechatronikerin erworben, ebenfalls stark vertreten waren (insbesondere in NRW) die Berufe Elektroniker und Elektronikerin für Energie- und Gebäudetechnik bzw. Elektroinstallateur und Elektroinstallateurin. Vereinzelt waren auch Ausbildungsberufe wie Kfz-Mechatroniker und -Mechatronikerin oder Elektroniker und Elektronikerin für Informations- und Telekommunikationstechnik vertreten (ebd.). Die Arbeitsdauer (nach der Ausbildung) lag im Mittel bei 3,6 Jahren (SD 3,4), wobei sich die Erfahrungen inhaltlich deutlich unterschieden (s. unten).

Die Fachwissenstests wurden im Anschluss an einschlägige Vorarbeiten (vgl. van Waveren und Nickolaus 2015), in welchen für Auszubildende gegen Ende der Ausbildung dreidimensionale Ausdifferenzierungen dokumentiert wurden, so ausgestaltet, dass für den Fall einer mehrdimensionalen Modellierung reliable Abschätzungen in den Subdimensionen zu erwarten waren. Die Modellprüfungen ergaben sowohl für den Eingangstest als auch den Abschlusstest erwartungskonform für das dreidimensionale Modell den besten Modellfit. Wie bei den Elektronikern und Elektronikerinnen für Automatisierungstechnik in vorangegangenen Studien werden die Wissensdimensionen Grundlagen der Elektrotechnik (ET), elektrische Anlagen und Systeme (EAS) und Steuerungstechnik (ST) als Subdimensionen des Fachwissens bestätigt (vgl. van Waveren und Nickolaus 2015; Nitzschke et al. 2017; Velten et al. 2018). Für den Test zeigen sich im Anschluss an die Testskalierung für den Eingangs- und Abschlusstest in allen Subdimensionen gute Reliabilitätswerte. ${ }^{5}$ Die analytische Problemlösekompetenz wurde über die im Handlungsfeld besonders leistungskritische Fähigkeit erfasst, in einem steuerungstechnischen System Fehler zu

\footnotetext{
5 Eingangstest: EAP/PV (WLE-Rel. $)=0,75(0,65) / 0,67(0,56) / 0,79(0,73)($ ET/EAS/ST); Abschlusstest: EAP/PV $($ WLE-Rel. $)=0,87(0,79) / 0,83(0,72) / 0,76(0,69)($ ET/EAS/ST $)$.
} 
diagnostizieren. Eingesetzt wurde dieses Instrument am Ende der Fortbildung, da in Vortests deutlich wurde, dass der Test zu Beginn der Fortbildung für den größten Teil der Fortbildungsteilnehmer zu schwer war. Darüber hinaus wurden auch die kognitiven Grundfähigkeiten (IQ) mit dem CFT 3 (Weiß 1971) erhoben, da unterstellt werden kann, dass deren Ausprägung auch die Problemlöseleistung beeinflusst.

Die inhaltlichen Arbeitserfahrungen wurden über eine Befragung der Fortbildungsteilnehmenden erfasst. Dazu wurden den angehenden staatlich geprüften Technikern und Technikerinnen zu Beginn der Fortbildung eine Liste mit insgesamt 22 Tätigkeiten vorgelegt, die bezogen auf die Häufigkeit der Ausführung auf einer sechsstufigen Skala von 1 ,nie“ bis 6 ,,sehr häufig“ einzuschätzen waren. Berücksichtigt wurden beispielsweise Items wie „mit elektrotechnischen Anlagen arbeiten“, „Maschinen warten und reparieren“, „Prozessabläufe analysieren“, „Prüf- und Messaufgaben planen und auswerten“, „Kostenkalkulationen und Abrechnungen durchführen“, „Team- und Kundenbesprechungen durchführen“ und „sich weiterbilden, lernen“. Auf Basis einer exploratorischen Faktorenanalyse (Hauptkomponentenanalyse mit Varimax-Rotation) wurden inhaltlich affine Tätigkeiten zu Subdimensionen zusammengefasst. Bezogen auf die Fehlerdiagnosekompetenz liegt es nahe, insbesondere die Subdimension „steuerungstechnische Erfahrungen“, die über die vier Tätigkeiten „Fehler in elektrotechnischen/steuerungstechnischen Anlagen finden“, „Maschinen warten und reparieren“, „Schaltgeräte und/oder Automatisierungssysteme montieren“ sowie „Systeme programmieren und konfigurieren“ gebildet wurde (Velten et al. 2018), als inhaltsaffin in den Blick zu nehmen.

Die Tests zur Erfassung der berufsfachlichen Kompetenzen wurden IRT basiert skaliert. Die Prüfung von $\mathrm{Hl}$ und $\mathrm{H} 2$ erfolgte über ein Strukturgleichungsmodell, zur Prüfung von $H 3$ wurden Korrelationsanalysen durchgeführt.

\section{Ergebnisse}

Angesichts der großen Unterschiede in den formalen Voraussetzungen war zu erwarten, dass auch erhebliche Unterschiede im Eingangswissen der Fortbildungsteilnehmenden beobachtet werden können. Im Anschluss an vorliegende Studien im Ausbildungsbereich, in welchen Auszubildende des Handwerks und der Industrie verglichen wurden, waren weiter deutliche Unterschiede zwischen diesen Gruppen zu erwarten, wobei zu berïcksichtigen ist, dass sich diese beiden Gruppen sowohl im Hinblick auf die allgemeinbildenden Abschlüsse, die kognitiven Grundfähigkeiten aber auch bezogen auf die inhaltlichen Tätigkeitsschwerpunkte unterscheiden.

Im Anschluss an die Studien im Ausbildungsbereich wurden für die Gruppe der Fortbildungsteilnehmenden Mittelwertvergleiche basierend auf dem Ausbildungshintergrund (Handwerk/Industrie) gerechnet. Hierbei wurden die Fachleistungen im Bereich des Problemlösens und des Fachwissens zu beiden Messzeitpunkten berücksichtigt. Aus Tab. 1 geht hervor, dass die auf den WLE Personenparametern basierenden Mittelwerte der Fachleistungen durchgängig und hochsignifikant zugunsten der Fortbildungsteilnehmer mit industriellem Hintergrund ausfallen.

In ergänzenden Analysen wurde deutlich, dass von den kognitiven Eingangsmerkmalen (kognitive Grundfertigkeit, Schulabschluss, Abschlussnote der Ausbildung) 
Tab. 1 Leistungsunterschiede von Fortbildungsschülern und -schülerinnen aus Handwerk und Industrie zu Beginn und am Ende der Fortbildung (Velten et al. 2018)

\begin{tabular}{|c|c|c|c|c|c|}
\hline & \multirow[b]{2}{*}{ Zeitpunkt } & \multicolumn{2}{|c|}{$\begin{array}{l}\text { Ausbildungshintergrund (Mit- } \\
\text { telwert, Standardabweichung } \\
(N))\end{array}$} & \multicolumn{2}{|l|}{ T-Test } \\
\hline & & Industrie & Handwerk & $\begin{array}{l}\text { T-Wert, } \\
p\end{array}$ & $\begin{array}{l}\text { Effekt- } \\
\text { größe d }\end{array}$ \\
\hline \multirow[t]{2}{*}{ Problemlösefähigkeit } & $\mathrm{t} 1$ & - & - & - & - \\
\hline & $\mathrm{t} 2$ & $\begin{array}{l}0,47,0,83 \\
(66)\end{array}$ & $\begin{array}{l}-0,64,1,33 \\
(37)\end{array}$ & $\begin{array}{l}4,56 \\
0,000\end{array}$ & 1.07 \\
\hline \multirow[t]{2}{*}{$\begin{array}{l}\text { Fachwissen - } \\
1 \text { Dimensional }\end{array}$} & $\mathrm{t} 1$ & $\begin{array}{l}0,43,0,79 \\
(90)\end{array}$ & $\begin{array}{l}-0,47,0,86 \\
(50)\end{array}$ & $\begin{array}{l}6,29 \\
0,000\end{array}$ & 1,10 \\
\hline & $\mathrm{t} 2$ & $\begin{array}{l}0,50,1,12 \\
(79)\end{array}$ & $\begin{array}{l}-0,66,1,04 \\
(45)\end{array}$ & $\begin{array}{l}5,67 \\
0,000\end{array}$ & 1,06 \\
\hline \multirow{2}{*}{$\begin{array}{l}\text { Fachwissen - } \\
\text { Dimension Grundlagen } \\
\text { der Elektrotechnik }\end{array}$} & $\mathrm{t} 1$ & $\begin{array}{l}0,42,1,26 \\
(90)\end{array}$ & $\begin{array}{l}-0,40,1,10 \\
(50)\end{array}$ & $\begin{array}{l}3,84 \\
0,000\end{array}$ & 0,68 \\
\hline & $\mathrm{t} 2$ & $\begin{array}{l}0,68,1,68 \\
(79)\end{array}$ & $\begin{array}{l}-0,96,1,47 \\
(45)\end{array}$ & $\begin{array}{l}5,48 \\
0,000\end{array}$ & 1,02 \\
\hline \multirow{2}{*}{$\begin{array}{l}\text { Fachwissen - } \\
\text { elektrische Anlagen und } \\
\text { Systeme }\end{array}$} & $\mathrm{t} 1$ & $\begin{array}{l}0,37,1,26 \\
(90)\end{array}$ & $\begin{array}{l}-0,29,1,16 \\
(50)\end{array}$ & $\begin{array}{l}3,041, \\
0,003\end{array}$ & 0,54 \\
\hline & $\mathrm{t} 2$ & $\begin{array}{l}0,51,1,33 \\
\text { (79) }\end{array}$ & $\begin{array}{l}-0,65,1,52 \\
(45)\end{array}$ & $\begin{array}{l}4,43 \\
0,000\end{array}$ & 0,83 \\
\hline \multirow[t]{2}{*}{$\begin{array}{l}\text { Fachwissen - } \\
\text { Steuerungstechnik }\end{array}$} & $\mathrm{t} 1$ & $\begin{array}{l}0,71,1,52 \\
(90)\end{array}$ & $\begin{array}{l}-0,71,1,25 \\
(50)\end{array}$ & $\begin{array}{l}5,64 \\
0,000\end{array}$ & 0,99 \\
\hline & $\mathrm{t} 2$ & $\begin{array}{l}0,32,1,07 \\
(79)\end{array}$ & $\begin{array}{l}-0,63,1,30 \\
(45)\end{array}$ & $\begin{array}{l}4,39 \\
0,000\end{array}$ & 0,82 \\
\hline
\end{tabular}

Angegeben sind die WLE Mittelwerte

bei Einbezug des Ausbildungshintergrunds lediglich die kognitiven Grundfähigkeiten und die Abschlussnote für das Eingangswissen erklärungsrelevant werden, besonders stark ist allerdings der Einfluss des Ausbildungshintergrunds (Handwerk, Industrie), der auch unterschiedliche Möglichkeiten bietet Berufserfahrungen zu sammeln. Die Berufserfahrung, gemessen über die Tätigkeitsdauer, erbringt keinen Beitrag zur Ausprägung des Fachwissens zu Beginn der Fortbildung, was so interpretiert werden kann, dass die Tätigkeitsdauer vor der Fortbildung allein weder negativ (im Sinne des Vergessens) noch positiv (im Sinne einer Fachwissenserweiterung) bedeutsam wird. Von besonderem Interesse ist vor dem Hintergrund der in Abschn. 1 eingebrachten Überlegungen und der entwickelten Hypothesen, ob die praktische Erfahrung bei einer tätigkeitsbezogenen Erfassung, statt einer Messung über die Ausbildungsdauer allein, doch noch erklärungsrelevant wird und bezogen auf welche Kompetenzbereiche gegebenenfalls Einflüsse beobachtet werden können. In Abb. 1 sind zentrale Ergebnisse der einschlägigen Analysen dargestellt.

Es ist erkennbar, dass sich die Arbeitserfahrungen im Bereich der Steuerungstechnik sowohl direkt auf die Ausprägung der Problemlöseleistung am Ende der Fortbildung auswirken als auch indirekt über das Fachwissen. D.h., die Ausprägung des Fachwissens zu Beginn der Technikerausbildung wird in den Subdimensionen der elektrischen Anlagen und Systeme und dem steuerungstechnischen Wissen signifikant durch die Arbeitserfahrung beeinflusst. Das Grundlagenwissen scheint 
A. Nitzschke et al.

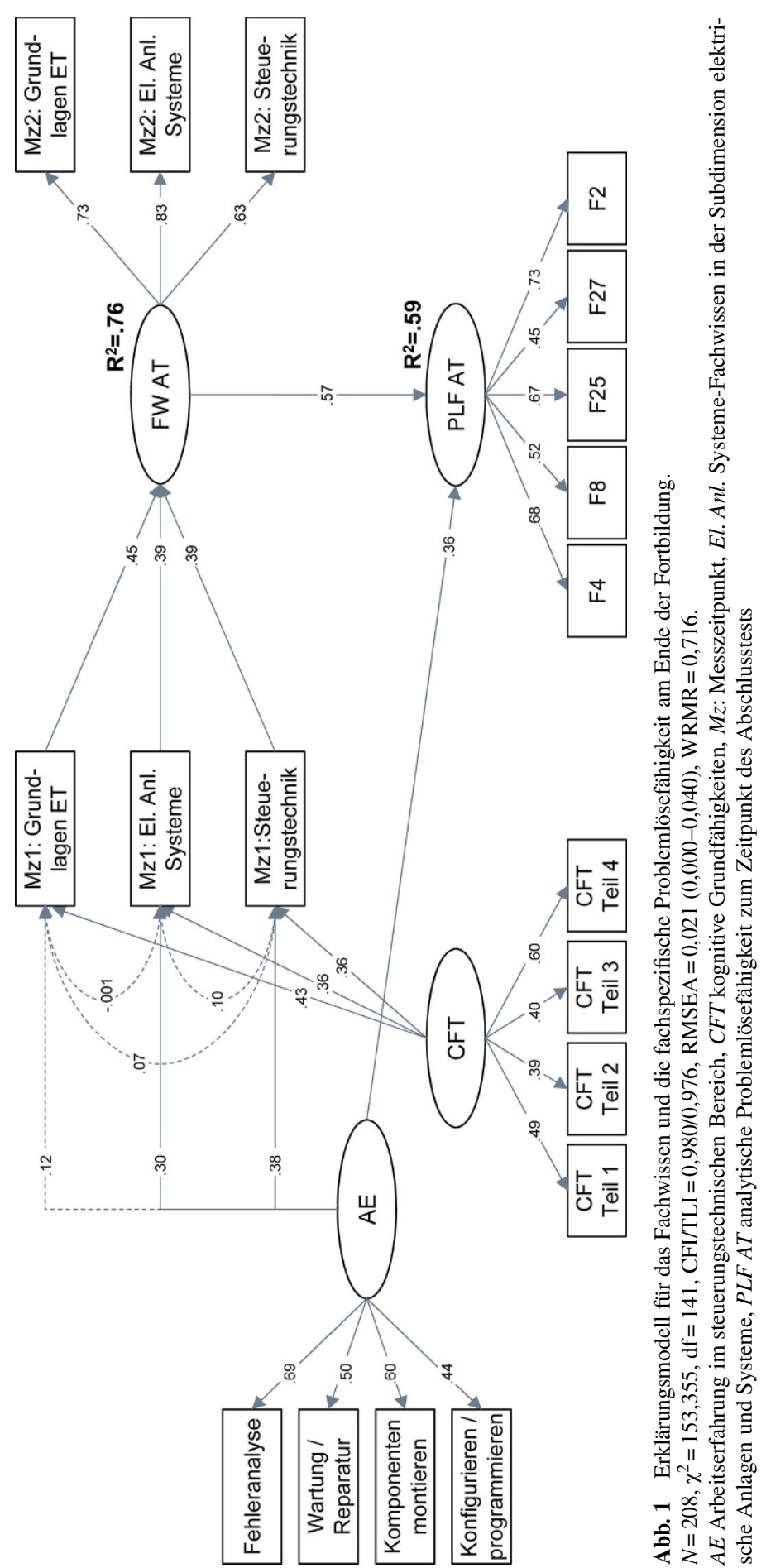


hingegen, wie in anderen Untersuchungen dokumentiert, primär durch die schulischen Lerngelegenheiten bestimmt (van Waveren und Nickolaus 2016). Damit wird H1 gestützt. Mitbestimmt ist das Fachwissen zu Beginn der Fortbildung auch von den kognitiven Grundfähigkeiten, die für das Grundlagenwissen etwas bedeutsamer werden, bei den beiden anderen Wissensdimensionen bleibt die berufliche Erfahrung nur wenig hinter der Erklärungskraft der kognitiven Grundfähigkeiten zurück. Die Ausprägungen des Fachwissens in den drei Subdimensionen zum Zeitpunkt des Eingangstests erklären die Varianz des Wissens zum Zeitpunkt des Abschlusstests mit einem sehr hohen Anteil von $76 \%$, wobei die Beiträge der einzelnen Subdimensionen ähnlich stark ausfallen. Hoch ist auch der erklärte Anteil der Problemlöseleistung durch das Fachwissen am Fortbildungsende. Die Ausprägungen des Fachwissens zu Beginn der Fortbildung werden nicht direkt, sondern nur indirekt über das Fachwissen am Fortbildungsende erklärungsrelevant. Bemerkenswert stark ist auch der Einfluss der steuerungstechnischen Erfahrungen auf die berufsfachlichen Problemlöseleistungen, womit auch $H 2$ gestützt wird. Zu berücksichtigen ist bei der Interpretation, dass die beruflichen Erfahrungen im Bereich der Steuerungstechnik auch stark durch den Ausbildungshintergrund (Industrie, Handwerk) bestimmt sind und in diese Bereiche auch bereits in die Ausbildung Personen mit stark unterschiedlichem Vorwissen einmünden. D.h., jene, die mehr Gelegenheiten haben, in diesem Segment berufliche Erfahrungen zu sammeln, hatten schon in frühen Phasen ihrer beruflichen Entwicklung Wissensvorteile gegenüber jenen, die in diesem Bereich weniger Erfahrungen vorweisen können. Bezieht man statt der Erfahrungen im steuerungstechnischen Bereich jene in anderen inhaltlichen distanten Themenfeldern heran, wie zum Beispiel die Erfahrungen im kaufmännischen Bereich (Controlling), so ergibt sich (erwartungskonform), wie bereits bei der Einbindung der Tätigkeitsdauer, keine Erklärungskraft dieses Erfahrungsbereiches für die Fehlerdiagnoseleistungen (Velten 2017).

Ergänzende Analysen, die zur Frage durchgeführt wurden, ob sich die Leistungsvorteile der Fortbildungsteilnehmenden mit industriellem Hintergrund im Laufe der Fortbildung abschwächen oder weiter verstärken, dokumentieren eher weitere Spreizungen als eine Leistungshomogenisierung (vgl. Tab. 1). D. h., die Leistungszuwächse sind bei den Leistungsstärkeren mit Ausnahme der Steuerungstechnik größer als bei den Leistungsschwächeren. Zu berücksichtigen ist bei der Interpretation dieser Ergebnisse, dass die Unterschiede nicht allein auf die Aus- und Fortbildung zurückzuführen sind, sondern durch Selektionsprozesse beim Übergang in die Ausbildung nach den vorliegenden Studien bereits deutliche Leistungsunterschiede zwischen den Auszubildenden in Industrie und Handwerk zugunsten der Auszubildenden der Industrie bestehen (vgl. z. B. Nickolaus et al. 2006).

Im Anschluss an Gespräche mit Lehrkräften, in welchen immer wieder auf die hohe Leistungsheterogenität bei der Einmündung in die Fortbildung und die Notwendigkeit, zunächst eine gemeinsame Ausgangsbasis zu schaffen, verwiesen wurde, wurde erwartet, dass sich eine Homogenisierung beobachten lässt. Das gilt in schwacher Form allerdings nur für die Wissensdimension der Steuerungstechnik, in der der Leistungsabstand zwischen Handwerk und Industrie zu t2 geringer ist als zu t1 (vgl. Tab. 1). Im Bereich der Grundlagen der Elektrotechnik und den elektrischen Anlagen und Systemen sind hingegen deutliche Zuwächse der Unterschiede 
beobachtbar. Für das eindimensional skalierte Fachwissen bleiben die Unterschiede in gleicher Größenordnung bestehen. Offensichtlich gelingt es nicht, die zum Teil bereits bei Beginn der Fortbildung sehr großen Leistungsunterschiede zu kompensieren, womit die Frage aufgeworfen wird, ob bei der Technikerausbildung ähnlich wie im Hochschulbereich Vorbereitungskurse notwendig werden, um auch jenen eine wünschenswerte Kompetenzentwicklung zu ermöglichen, die mit ungünstigeren Voraussetzungen in die Fortbildung einmünden.

Die Prüfung von $H 3$ zu einer zunehmend integrativen Verarbeitung des Fachwissens stützt die eingebrachte Annahme, dass die Wissensbereiche gegen Ende der Fortbildung stärker korrelieren und damit integrativer verarbeitet werden. Wir schließen das aus wesentlich engeren Zusammenhängen der Fachwissensdimensionen am Ende der Fortbildung als zu deren Beginn. Die Korrelationen zwischen den Subdimensionen im Eingangstest liegen im Bereich von 0,34 bis 0,53, im Abschlusstest werden deutlich höhere Zusammenhänge dokumentiert (0,60 bis 0,82$)$. Besonders groß wird im Abschlusstest die Korrelation zwischen den Grundlagen der Elektrotechnik und der Subdimension elektrische Anlagen und Systeme $(r=0,82)$. Das ist insoweit vorteilhaft, als eine möglichst tiefe Durchdringung des relevanten Wissens, die u. a. auch in der (stärkeren) Verknüpfung der Wissensbereiche Ausdruck findet, auch die Anwendungsfähigkeit des Fachwissens begünstigt.

\section{Diskussion}

Die Ergebnisse zeigen sowohl die Bedeutung spezifischer tätigkeitsbezogen erhobener berufsfachlicher Erfahrungen für den Fachwissenserwerb als auch für die fachliche Problemlösefähigkeit. Bemerkenswert ist insbesondere der relativ starke direkte Einfluss der inhaltlich affinen Erfahrungsbereiche auf die Fehlerdiagnosekompetenz. Dass den beruflichen Erfahrungen in anderen Tätigkeitssegmenten und der Tätigkeitsdauer allein keine Bedeutung für die Ausprägung der Fehlerdiagnosekompetenz zukommt, stützt letztlich die inhaltliche Interpretation eines kausalen Zusammenhangs zwischen den einschlägigen beruflichen Erfahrungen und den fachlichen Leistungen. Die in den Metastudien dokumentierten schwachen Einflüsse der beruflichen Erfahrungen auf die berufliche Performanz scheint der unspezifischen Erfassung der beruflichen Erfahrungen über die Tätigkeitsdauer, d.h. dem verwendeten Kriterium geschuldet. Zugleich wird allerdings mit den Ergebnissen nochmals deutlich, dass höherwertige berufsfachliche Leistungen, wie im Bereich der Fehlerdiagnose, in hohem Grade an die Verfügbarkeit des einschlägigen Fachwissens gebunden sind. In ergänzenden Analysen (vgl. Velten et al. 2018) wurde wie in anderen Studien (Nickolaus et al. 2016; Walker et al. 2016) deutlich, dass vor allem jene Fachwissensbereiche für die Fehlerdiagnoseleistungen erklärungsrelevant werden, die auch inhaltlich enge Bezüge zur Fehlerdiagnose besitzen. Das Grundlagenwissen wird dafür nicht erklärungsrelevant, ist allerdings notwendig für den Aufbau des fachlichen Spezialwissens, wie es in den anderen Subdimensionen des Fachwissens Ausdruck findet.

Relevant sind diese Ergebnisse u.E. auch für die Anerkennungspraxis, was beispielsweise die Anerkennung außerinstitutionell erworbener Kompetenzen oder 
nicht formaler Qualifikationen betrifft. Wenn das Fachwissen die höchste Erklärungskraft für die (anspruchsvollen) berufsfachlichen Leistungen besitzt, spricht viel dafür, mit relativ geringem Aufwand dieses Fachwissen auch zu testen. Vermieden werden sollten Kompetenzzuschreibungen, die allein auf der Tätigkeitsdauer in einem Tätigkeitsbereich beruhen. Wesentlich aussagekräftiger sind dafür die spezifischen Tätigkeitserfahrungen, d.h. inhaltlich möglichst konkret erhobene Berufserfahrungen. Angesichts dessen, dass allein mit diesen Variablen (Fachwissen und spezifische berufliche Erfahrungen) ca. $60 \%$ der Varianz der Fehlerdiagnoseleistung vorhergesagt werden kann, bietet dieser kombinierte Ansatz eine praktikable Möglichkeit, eine relativ gute Abschätzung des Leistungsvermögens vorzunehmen. In einem alternativ gerechneten Modell, in welchem lediglich die spezifischen Berufserfahrungen unter Kontrolle der kognitiven Leistungsfähigkeit als Prädiktoren berücksichtigt wurden, ist die Prognosekraft zwar deutlich geringer, liegt mit einer erklärten Varianz von $32 \%$ jedoch über dem, was mit einer Beschränkung auf rein formale Kriterien wie der Dauer der Berufsausübung erreicht werden kann. Anzumerken ist, dass für die Erfassung der beruflichen Erfahrungen bisher nur relativ grob gefasste Tätigkeitsfelder verwendet wurden und daher davon ausgegangen werden kann, dass sich die Erklärungskraft durch eine detailliertere Erfassung der beruflichen Erfahrungen weiter steigern lässt. Arbeitsproben wären sicherlich noch aussagekräftiger, aber auch wesentlich aufwändiger und würden vermutlich kaum realisiert.

Wichtig sind aus einer praktischen Perspektive auch die Ergebnisse zu den großen Leistungsunterschieden der Fortzubildenden, die im Laufe der Fortbildung eher noch anwachsen, als dass sie ausgeglichen werden könnten. Deutliche Leistungsunterschiede zeigen sich insbesondere zwischen Fortbildungsschülern und -schülerinnen mit handwerklichem und industriellem Hintergrund. Der Umstand, dass diese bereits zu Beginn der Fortbildung bestehen und es im Laufe der Fortbildung zu keiner Homogenisierung kommt, zeigt, dass es derzeit nicht durchweg gelingt, Leistungsdefizite, insbesondere bei Leistungsschwächeren, im Verlauf der beruflichen Qualifikation abzubauen. Möglichkeiten, dem entgegenzuwirken, ergeben sich beispielsweise durch eine stärkere Fokussierung auf lernförderliche Lern- und Arbeitsbedingungen oder den Ausbau individueller Fördermaßnahmen. Denkbar wären für den Ausgleich bzw. die Milderung der Leistungsunterschiede und die Schaffung einer günstigeren Startposition für die Leistungsschwächeren sowohl an den Schulen einzurichtende Vorkurse als auch digital gestützte Lernprogramme, die auf die Absicherung eines Mindestwissens ausgerichtet werden und in Blended Learning Scenarios eingebracht werden könnten. Begünstigt scheint die hohe Heterogenität auch dadurch, dass unter spezifischen Bedingungen auch Bewerber und Bewerberinnen aufgenommen werden, die aufgrund ihrer Bildungsbiographie nur bedingt anschlussfähig sind. Auch wenn angenommene Bewerber und Bewerberinnen mit nur gering affinen Abschlüssen wie beispielsweise Technische Zeichner eher die Ausnahme darstellen, stellt sich die Frage, ob solche Aufnahmepraxen zielführend sind. Bezogen auf die fachlichen Kompetenzen und beruflichen Erfahrungen gehen wir davon aus, dass sich, insbesondere im beruflichen Bereich, in weiteren Fortbildungsprogrammen ähnliche Ausgangslagen zeigen. Interessant wären in diesem 
Zusammenhang Studien, welche im Rahmen einer Intervention, Fördermaterialen basierend auf den beruflichen Erfahrungen bereitstellen.

Die Ergebnisse zur Prüfung von $H 3$ machen deutlich, dass es im Zuge der Fortbildung zu einer engeren Assoziation der Fachwissensdimensionen kommt, wofür wir eine stärkere integrative Verarbeitung der Wissensbereiche als ursächlich vermuten, was auch eine Erhöhung der Anwendungsfähigkeit des Fachwissens erwarten lässt.

Limitationen der Studie ergeben sich einerseits durch die Beschränkung auf eine Domäne, die Beschränkung auf die Vollzeitvarianten der Technikerschule, die Beschränkung auf die Erfassung der analytischen Problemlöseleistungen bzw. den Verzicht auch konstruktive Problemlöseleistungen einzubeziehen sowie die relativ kleine Stichprobe, welche lediglich aus zwei Bundesländern stammt. Limitierend sind andererseits die im Verlaufe der Studie deutlich gewordenen Probleme, zum Fortbildungsbeginn verfügbare Testinstrumentarien zur Erfassung der berufsfachlichen Problemlösekompetenzen einzusetzen. Ursächlich war dafür die stark ausgeprägte Heterogenität der Probandengruppe, die für große Anteile der Testpersonen zu Beginn der Fortbildungsmaßnahme Bodeneffekte zur Folge hatte und zum Abbruch des einschlägigen Tests führten. Während bezogen auf das Fachwissen trotz der stark unterschiedlichen Vorbildungen hinreichende Überschneidungen der curricularen Basen bestehen, scheinen die berufsfachlichen Problemlöseleistungen doch stärker durch die jeweilige Fachrichtung geprägt. Nachzugehen wäre in weiteren Analysen auch der Frage nach den erreichten Leistungsniveaus und den Fortbildungsbedingungen, die für die Leistungsentwicklung bedeutsam werden.

Open Access Dieser Artikel wird unter der Creative Commons Namensnennung 4.0 International Lizenz (http://creativecommons.org/licenses/by/4.0/deed.de) veröffentlicht, welche die Nutzung, Vervielfältigung, Bearbeitung, Verbreitung und Wiedergabe in jeglichem Medium und Format erlaubt, sofern Sie den/die ursprünglichen Autor(en) und die Quelle ordnungsgemäß nennen, einen Link zur Creative Commons Lizenz beifügen und angeben, ob Änderungen vorgenommen wurden.

Publisher's Note Springer Nature remains neutral with regard to jurisdictional claims in published maps and institutional affiliations.

\section{Literatur}

Abele, S., Behrendt, S., Weber, W., \& Nickolaus, R. (2015). Berufsfachliche Kompetenzen von Kfz Mechatronikern (Verbundprojekt KOKO Kfz). In BMBF (Hrsg.), Technologiebasierte Kompetenzmessung in der beruflichen Bildung (ASCOT). Ergebnisse und Bedeutung für Politik und Praxis (S. 20-23). Bonn: BMBF.

Dette, D.E., Abele, A.E., \& Renner, O. (2004). Zur Definition und Messung von Berufserfolg - Theoretische Überlegungen und metaanalytische Befunde zum Zusammenhang von externen und internen Laufbahnerfolgsmaßen. Zeitschrift für Personalpsychologie, 3, 170-183.

Dye, D. A., Reck, M., \& McDaniel, M. A. (1993). The validity of job knowledge measures. International Journal of Selection and Assessment, 1(3), 153-157.

Geithner, E., \& Moser, K. (2009). Die Messung von herausforderungsbasierter Berufserfahrung. In D. Münk \& E. Severin (Hrsg.), Theorie und Praxis der Kompetenzfeststellung im Betrieb - Status quo und Entwicklungsbedarf (S. 70-91). Bonn: Bundesinstitut für berufliche Bildung.

Hunter, J., \& Hunter, R. (1984). Validity and utility of alternative predictors of job performance. Psychological Bulletin, 96, 72-98.

Ng, T. W.H., Eby, L. T., Sorensen, K.L., \& Feldman, D.C. (2005). Predictors of objective and subjective career success: a meta-analysis. Personnel Psychology, 58(2), 367-408. 
Nickolaus, R., \& Seeber, S. (2013). Berufliche Kompetenzen: Modellierungen und diagnostische Verfahren. In A. Frey, U. Lissmann \& B. Schwarz (Hrsg.), Handbuch Berufspädagogische Diagnostik (S. 166-195). Weinheim: Beltz.

Nickolaus, R., Behrendt, S., \& Abele, S. (2016). Kompetenzstrukturen bei KFZ-Mechatronikern und die Erklärungskraft des fachsystematischen Wissens für berufsfachliche Kompetenzen. Unterrichtswissenschaft, 22, 114-130.

Nickolaus, R., Knöll, B., \& Gschwendtner, T. (2006). Methodische Präferenzen und ihre Effekte auf die Kompetenz- und Motivationsentwicklung - Ergebnisse aus Studien in anforderungsdifferenten elektrotechnischen Ausbildungsberufen in der Grundbildung. Zeitschrift für Berufs- und Wirtschaftspädagogik, 102(4), 552-577.

Nitzschke, A., Velten, S., Dietzen, A., \& Nickolaus, R. (2017). Motive, Vorerfahrungen und kognitive Eingangsvoraussetzungen von Technikerschülern und Technikerschülerinnen. Zeitschrift für Berufsund Wirtschaftspädagogik, 113(3), 490-516.

Quiñones, M., Ford, K., \& Teachout, M. (1995). The relationship between work experience and job performance: a conceptual and mega-analytic review. Personnel Psychology, 48(4), 887-910.

Schmidt, F.L., \& Hunter, J.E. (1998). The validity and utility of selection methods in personnel psychology: practical and theoretical implications of 85 years of research findings. Psychological Bulletin, $124,262-274$.

Schrader, F. (2012). Lehrer als Diagnostiker. In E. Terhart, H. Bennewitz \& M. Rothland (Hrsg.), Handbuch der Forschung zum Lehrerberuf (S. 683-689). Münster: Waxmann.

Sonnentag, S., Volmer, J., \& Spychala, A. (2008). Job Performance. In J. Barling \& C.L. Cooper (Hrsg.), The SAGE handbook of organizational behavior (S. 427-447). Los Angeles, London: SAGE.

Tesluk, P.E., \& Jacobs, R. R. (1998). Toward an integrated model of work experience. Personnel Psychology, 51(2), 321-355.

Velten, S. (2017). What Affects Vocational Competencies in Post-secondary VET? International Journal for Cross-Disciplinary Subjects in Education, 8(2), 3091-3102.

Velten, S., Nitzschke, A., Nickolaus, R., \& Walker, F. (2018). Die Fachkompetenzstruktur von Technikern für Elektrotechnik und Einflussfaktoren auf ihre Kompetenzentwicklung. Journal of Technical Education, 6(1), 201-222.

Walker, F., Link, N., \& Nickolaus, R. (2015). Berufsfachliche Kompetenzstrukturen bei Elektronikern für Automatisierungstechnik am Ende der Berufsausbildung. Zeitschrift für Berufs- und Wirtschaftspädagogik, 111(2), 222-241.

Walker, F., Link, N., \& Nickolaus, R. (2016). A multidimensional structure of domain-specific problemsolving competencies of electronics technicians for automation technology. Empirical Research in Vocational Education and Training. https://doi.org/10.1186/s40461-016-0034-z.

van Waveren, L., \& Nickolaus, R. (2015). Struktur- und Niveaumodell des Fachwissens bei Elektronikern für Automatisierungstechnik. Journal of Technical Education, 3(2), 62-91.

van Waveren, L., \& Nickolaus, R. (2016). Einflüsse der Vermittlungsintensität von Schule und Betrieb auf das Fachwissen bei Elektronikern für Automatisierungstechnik. Zeitschrift für Berufs- und Wirtschaftspädagogik, 112(3), 382-405.

Weiß, R. H. (1971). Grundintelligenztest CFT 3. Braunschweig: Hogrefe. 